\title{
Infinite Iterated Function Systems: A Multivalued Approach
}

by

\author{
K. LEŚNIAK
}

Presented by Andrzej LASOTA

\begin{abstract}
Summary. We prove that a compact family of bounded condensing multifunctions has bounded condensing set-theoretic union. Compactness is understood in the sense of the Chebyshev uniform semimetric induced by the Hausdorff distance and condensity is taken w.r.t. the Hausdorff measure of noncompactness. As a tool, we present an estimate for the measure of an infinite union. Then we apply our result to infinite iterated function systems.
\end{abstract}

1. The space of multifunctions. Let $(X, d)$ be a complete metric space, and let $x_{0} \in X, r>0, A, B \subset X$. We denote by $B\left(x_{0}, r\right)$ the open $r$-ball at $x_{0}$, by $\mathcal{O}_{r}(A)=\bigcup_{a \in A} B(a, r)$ the $r$-neighbourhood of $A$, and by

$$
h(A, B)=\inf \left\{r>0: A \subset \mathcal{O}_{r}(B), B \subset \mathcal{O}_{r}(A)\right\}
$$

the Hausdorff semimetric. Moreover, we denote by $2^{X}$ the family of all nonempty subsets of $X$, and by $\mathcal{B}(X)$ the family (or hyperspace) of nonempty bounded subsets of $X$ equipped with the Hausdorff semimetric $h$. The symbol $\varphi: X \multimap X$ will stand for a multifunction with nonempty bounded values. Such a $\varphi$ can be identified with a mapping $\varphi: X \rightarrow \mathcal{B}(X)$. The image of $A$ under $\varphi$ is the set $\varphi(A)=\bigcup_{a \in A} \varphi(a)$. The set-theoretic union of multifunctions $\varphi_{t}: X \multimap X, t \in T$, is $\bigcup_{t \in T} \varphi_{t}: X \multimap X,\left(\bigcup_{t \in T} \varphi_{t}\right)(x)=$ $\bigcup_{t \in T} \varphi_{t}(x)$ for $x \in X$. For basic concepts of set-valued analysis see e.g. [HP].

2000 Mathematics Subject Classification: 54H25, 47H10, 47H09, 37B99.

Key words and phrases: compactness in Chebyshev's semimetric, Hausdorff measure of noncompactness, condensing multifunction, uniformly Hausdorff upper semicontinuous multifunction, iterated function system, Barnsley-Hutchinson operator, maximal fixed point, attractor. 
Let $Z$ be a nonempty set. We introduce the following spaces:

$$
\begin{aligned}
& \mathcal{B M}(X, X)=\{\varphi: X \multimap X: \varphi(X) \text { is bounded in } X\}, \\
& \mathcal{B}(Z, \mathcal{B}(X))=\left\{\varphi: Z \rightarrow \mathcal{B}(X):\{\varphi(z)\}_{z \in Z} \text { is bounded in } \mathcal{B}(X)\right\} .
\end{aligned}
$$

The second space is furnished with the Chebyshev semimetric

$$
h_{\sup }\left(\varphi_{1}, \varphi_{2}\right)=\sup _{z \in Z} h\left[\varphi_{1}(z), \varphi_{2}(z)\right]
$$

for $\varphi_{1}, \varphi_{2}: Z \rightarrow \mathcal{B}(X)$. We identify $\mathcal{B}(X)=\mathcal{B}(\star, \mathcal{B}(X))$ for $\star$ a singleton and $\mathcal{B M}(X, X)=\mathcal{B}(X, \mathcal{B}(X))$. In particular $\mathcal{B} \mathcal{M}(X, X)$ is equipped with the Chebyshev semimetric $h_{\text {sup }}$. Moreover, it is not hard to see the following:

Lemma 1. If $\varphi_{1}, \varphi_{2}: X \multimap X$, then

$$
\sup _{x \in X} h\left[\varphi_{1}(x), \varphi_{2}(x)\right]=\sup _{\emptyset \neq A \subset X} h\left[\varphi_{1}(A), \varphi_{2}(A)\right] .
$$

Therefore, we have

Proposition 1. The map $j: \mathcal{B M}(X, X) \rightarrow \mathcal{B}\left(2^{X}, \mathcal{B}(X)\right),[j(\varphi)](A)=$ $\varphi(A)$ for all $\varphi \in \mathcal{B M}(X, X)$ and $A \in 2^{X}$, is an isometric embedding, i.e. it is an injection preserving the semimetric $h_{\text {sup }}$.

The operation $j$ is called the united extension (see [W]).

Lemma 2. Let $X_{1}, X_{2}$ be semimetric spaces, with $X_{1}$ isometrically embedded in $X_{2}$ via $j: X_{1} \rightarrow X_{2}$. Then $A \subset X_{1}$ is (pre) compact if and only if $j(A) \subset X_{2}$ is.

Let $\Phi \subset \mathcal{B M}(X, X)$ and let $j$ be as in Proposition 1 . Then, by Lemma 2, the family $\Phi$ is (pre)compact in $\mathcal{B M}(X, X)$ iff $j(\Phi)$ is (pre)compact in $\mathcal{B}\left(2^{X}, \mathcal{B}(X)\right)$. Thus we can speak about $h_{\text {sup }}$-(pre)compactness with no ambiguity.

2. Hausdorff measure of noncompactness. We recall that the Hausdorff measure of noncompactness on a (semi)metric space $X$ is the functional $\beta: 2^{X} \rightarrow[0, \infty]$ defined by

$$
\beta(A)=\inf \left\{r>0: \exists x_{1}, \ldots, x_{k} \in X, \bigcup_{i=1}^{k} B\left(x_{i}, r\right) \supset A\right\} .
$$

Notice that $\beta(A)=\infty$ if and only if $A$ is unbounded. In the case of the hyperspace $\mathcal{B}(X)$ we shall write $B^{\#}(Z, \nu)=\{A \in \mathcal{B}(X): h(A, Z)<\nu\}$ for the open $\nu$-ball with center $Z \in \mathcal{B}(X)$, and $\beta^{\#}: 2^{\mathcal{B}(X)} \rightarrow[0, \infty]$ for the Hausdorff measure of noncompactness. More on measures of noncompactness can be found in [AKPRS].

We have the following (cf. [CV, Remark after Theorem II-4, p. 41] and [D, Chapt. 2, Sect. 7.4, pp. 42-43]): 
Lemma 3 (Estimate for infinite unions). If $\left\{A_{t}\right\}_{t \in T} \subset \mathcal{B}(X)$, then

$$
\sup _{t \in T} \beta\left(A_{t}\right) \leq \beta\left(\bigcup_{t \in T} A_{t}\right) \leq \sup _{t \in T} \beta\left(A_{t}\right)+2 \beta^{\#}\left(\left\{A_{t}\right\}_{t \in T}\right) .
$$

Proof. Let $\nu>\beta^{\#}\left(\left\{A_{t}\right\}_{t \in T}\right), \sigma>\sup _{t \in T} \beta\left(A_{t}\right)$. Then there exists a finite family $\left\{Z_{i}\right\}_{i=1}^{k} \subset \mathcal{B}(X)$ such that $\bigcup_{i=1}^{k} B^{\#}\left(Z_{i}, \nu\right) \supset\left\{A_{t}\right\}_{t \in T}$. Decompose $T=\bigcup_{i=1}^{k} T_{i}$, where $T_{i}=\left\{t \in T: A_{t} \in B^{\#}\left(Z_{i}, \nu\right)\right\}$. For $t \in T_{i}$ we have $Z_{i} \subset \mathcal{O}_{\nu}\left(A_{t}\right), A_{t} \subset \mathcal{O}_{\nu}\left(Z_{i}\right)$. Further, pick $t_{i} \in T_{i}$ in each $T_{i}$. Every $A_{t_{i}}$ can be covered by balls, $A_{t_{i}} \subset \bigcup_{j \in J(i)} B\left(x_{j}^{i}, \sigma\right)$, with $J(i)$ finite. As a result we obtain

$$
\begin{aligned}
& Z_{i} \subset \mathcal{O}_{\nu}\left(A_{t_{i}}\right) \subset \bigcup_{j \in J(i)} B\left(x_{j}^{i}, \nu+\sigma\right), \\
& A_{t} \subset \mathcal{O}_{\nu}\left(Z_{i}\right) \subset \bigcup_{j \in J(i)} B\left(x_{j}^{i}, \sigma+2 \nu\right) .
\end{aligned}
$$

Since $\nu, \sigma$ were arbitrary, the desired inequality follows.

Hence we infer a generalization of the well known equality $\beta\left(A_{1} \cup A_{2}\right)=$ $\max \left\{\beta\left(A_{1}\right), \beta\left(A_{2}\right)\right\}$.

Proposition 2. If $\left\{A_{t}\right\}_{t} \subset \mathcal{B}(X)$ is an h-precompact family, then

$$
\beta\left(\bigcup_{t} A_{t}\right)=\sup _{t} \beta\left(A_{t}\right) .
$$

3. Uniform Hausdorff upper semicontinuity. This section deals with the notion of continuity introduced in [L2]. We say that a multifunction $\varphi: X \multimap X$ is uniformly Hausdorff upper semicontinuous if for each $\varepsilon>0$ and each closed subset $A$ of $X$,

$$
\varphi\left[\mathcal{O}_{\delta} A\right] \subset \mathcal{O}_{\varepsilon} \varphi(A) \quad \text { for some } \delta>0 .
$$

This type of continuity appears in Section 5 (for more details see [L2]; nontrivial examples can be found in [HP] among those concerned with the differences between upper semicontinuity and Hausdorff upper semicontinuity).

TheOREM 1. Let $\left\{\varphi_{t}: X \multimap X\right\}_{t \in T}$ be an $h_{\text {sup-precompact family of }}$ uniformly Hausdorff upper semicontinuous multifunctions. Then $\bigcup_{t \in T} \varphi_{t}$ : $X \multimap X$ is again uniformly Hausdorff upper semicontinuous.

Proof. Fix $\varepsilon>0$ and closed $A \subset X$. By $h_{\text {sup }}$-precompactness choose a finite $\varepsilon / 3$-net $\left\{\varphi_{t_{i}}\right\}_{i=1}^{k}$, i.e., for each $t$ there is $i$ such that

$$
h_{\sup }\left(\varphi_{t}, \varphi_{t_{i}}\right)<\varepsilon / 3 \text {. }
$$


Since $\varphi_{t_{1}}, \ldots, \varphi_{t_{k}}$ are uniformly Hausdorff upper semicontinuous, for each $i$ there is $\delta_{t_{i}}>0$ such that $\varphi_{t_{i}}\left[\mathcal{O}_{\delta_{t_{i}}}(A)\right] \subset \mathcal{O}_{\varepsilon / 3} \varphi_{t_{i}}(A)$. Hence

$$
\begin{aligned}
\varphi_{t}\left[\mathcal{O}_{\delta_{t_{i}}}(A)\right] & \subset \mathcal{O}_{\varepsilon / 3} \varphi_{t_{i}}\left[\mathcal{O}_{\delta_{t_{i}}}(A)\right] \subset \mathcal{O}_{\varepsilon / 3} \mathcal{O}_{\varepsilon / 3}\left[\varphi_{t_{i}}(A)\right] \\
& \subset \mathcal{O}_{3 \varepsilon / 3} \varphi_{t}(A) \subset \mathcal{O}_{\varepsilon}\left[\bigcup_{t} \varphi_{t}(A)\right] .
\end{aligned}
$$

Putting $\delta=\min \left\{\delta_{t_{i}}: i=1, \ldots, k\right\}$ we get $\bigcup_{t} \varphi_{t}\left[\mathcal{O}_{\delta}(A)\right] \subset \mathcal{O}_{\varepsilon}\left[\bigcup_{t} \varphi_{t}(A)\right]$.

Similarly to Theorem 1 one can prove

Proposition 3. The subspace of $\mathcal{B M}(X, X)$ consisting of bounded uniformly Hausdorff upper semicontinuous multifunctions is closed in the $h_{\text {sup }}$ semimetric.

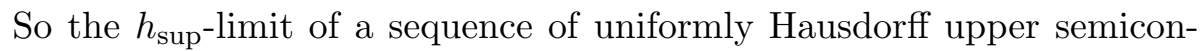
tinuous maps is again a map of the same kind.

4. Compact families of condensing maps. We say that a multifunction $\varphi: X \multimap X$ is:

- a $\beta$-contraction if there exists $L<1$ such that

$$
\beta[\varphi(A)] \leq L \cdot \beta(A)
$$

for all $A \subset X$ with $\beta(A)<\infty$;

- a $\beta$-condensing map if

$$
\beta[\varphi(A)]<\beta(A)
$$

for all $A \subset X$ with $\beta(A)<\infty$ and $\beta[\varphi(A)]>0$.

Observe that every $\beta$-contraction is $\beta$-condensing.

We define, for every $A \subset X$, the evaluation map ev $\operatorname{ev}_{A}: \mathcal{B M}(X, X) \rightarrow$ $\mathcal{B}(X)$ by $\operatorname{ev}_{A}(\varphi)=\varphi(A)$ for all $\varphi \in \mathcal{B M}(X, X)$. From Lemma 1 we obtain:

Lemma 4. For every $A \subset X$ the evaluation map $\operatorname{ev}_{A}: \mathcal{B M}(X, X) \rightarrow$ $\mathcal{B}(X)$ is nonexpansive, i.e.,

$$
h\left[\operatorname{ev}_{A}\left(\varphi_{1}\right), \operatorname{ev}_{A}\left(\varphi_{2}\right)\right] \leq h_{\text {sup }}\left(\varphi_{1}, \varphi_{2}\right) .
$$

Recall that the Hausdorff measure of noncompactness $\beta: \mathcal{B}(X) \rightarrow[0, \infty)$ is nonexpansive, i.e., $\left|\beta\left(A_{1}\right)-\beta\left(A_{2}\right)\right| \leq h\left(A_{1}, A_{2}\right)$; so, in particular, it is continuous (e.g. [AKPRS]). Now we can state the main result.

Theorem 2 (on compact unions of condensing maps). Let

$$
\left\{\varphi_{t}: X \multimap X\right\}_{t \in T} \subset \mathcal{B M}(X, X)
$$

be an $h_{\text {sup }}$-compact family of bounded $\beta$-condensing multifunctions. Then $\bigcup_{t \in T} \varphi_{t}: X \multimap X$ is also a bounded $\beta$-condensing map. 
Proof. Boundedness is obvious (just take a finite net of multifunctions). To verify $\beta$-condensity, fix $A \subset X$ with $\beta(A)<\infty$. Since $\left\{\varphi_{t}\right\}_{t \in T}$ is $h_{\text {sup }^{-}}$ compact, the family $\left\{\varphi_{t}(A)\right\}_{t \in T} \subset \mathcal{B}(X)$ is $h$-compact (Lemma 4). By continuity of $\beta$ the function $t \mapsto \beta\left[\varphi_{t}(A)\right]$ attains its maximum at some $t_{0}$. Finally,

$$
\beta\left[\bigcup_{t \in T} \varphi_{t}(A)\right]=\sup _{t \in T} \beta\left[\varphi_{t}(A)\right]=\beta\left[\varphi_{t_{0}}(A)\right]<\beta(A)
$$

when the left hand side is $>0$; the first equality follows from Proposition 2.

As one might expect, compactness cannot be weakened to precompactness in Theorem 2. More exactly, an $h_{\text {sup }}$-precompact family of $\beta$-contractions need not have a $\beta$-condensing union. To see this, simply take the radial projection $\varrho$ onto the closed unit ball in an infinite-dimensional Banach space and the family $\{L \cdot \varrho\}_{0 \leq L<1}$ of $\beta$-contractive maps.

However, there still remains an open question: does the $h_{\text {sup-compact }}$ family of $\beta$-contractions have a $\beta$-contractive union? We only make some observation in this direction.

One can associate with a multifunction $\varphi: X \multimap X$ its $\beta$-contractivity constant

$$
L(\varphi)=\inf \{L>0: \beta[\varphi(A)] \leq L \cdot \beta(A) \text { for all } A \subset X\} .
$$

It has the following property:

Proposition 4. The extended real valued function $\mathcal{B M}(X, X) \ni \varphi \mapsto$ $L(\varphi) \in[0, \infty]$ is lower semicontinuous.

Proof. Let $h_{\text {sup }}\left(\varphi_{n}, \varphi\right) \rightarrow 0$ as $n \rightarrow \infty$ and $A \subset X$ be bounded, i.e. $\beta(A)<\infty$. For every $\varepsilon>0$ there exists $m$ such that $\varphi(A) \subset \mathcal{O}_{\varepsilon} \varphi_{n}(A)$ for all $n \geq m$. Therefore

$$
\begin{aligned}
& \beta[\varphi(A)] \leq \inf _{n \geq m} \beta\left[\varphi_{n}(A)\right]+\varepsilon \leq \inf _{n \geq m} L\left(\varphi_{n}\right) \cdot \beta(A)+\varepsilon, \\
& \beta[\varphi(A)] \leq \sup _{m} \inf _{n \geq m} L\left(\varphi_{n}\right) \cdot \beta(A) ;
\end{aligned}
$$

so $L(\varphi) \leq \liminf _{n \rightarrow \infty} L\left(\varphi_{n}\right)$, which shows the lower semicontinuity (see e.g. $[\mathrm{HP}])$.

Thus every $h_{\text {sup }}$-compact family of $\beta$-contractions contains one with the minimal $\beta$-contractivity constant. We do not know any counterexample to the conjecture that the maximal constant is also attained.

5. Application to iterated function systems. Iterated function systems (IFS) have been extensively studied at various levels of generality ([Hu], $[\mathrm{SV}],[\mathrm{Ha}],[\mathrm{JGP}],[\mathrm{H}],[\mathrm{AF}],[\mathrm{AG}],[\mathrm{W}],[\mathrm{E}],[\mathrm{LM}],[\mathrm{K}])$. Two streams of research could be singled out: set-theoretical and topological. However, such 
divisions would be unsuitable, because of the natural interplay between order and topology (see $[\mathrm{CoV}]$ ).

Below we collect some necessary definitions from [L1] and [L2]. A family $\left\{\varphi_{t}: X \multimap X\right\}_{t \in T}$ of multifunctions is said to be a multivalued iterated function system, and the operator $F: 2^{X} \rightarrow 2^{X}, F(A)=\overline{\bigcup_{t \in T} \varphi_{t}(A)}$ for $A \in 2^{X}$, is called its Barnsley-Hutchinson operator. In the case of a finite family $\left\{\varphi_{1}, \ldots, \varphi_{k}: X \multimap X\right\}$ one can always replace it with a singleton $\{\varphi: X \multimap X\}$, where $\varphi(x)=\bigcup_{i=1}^{k} \varphi_{i}(x)$. Many properties of multifunctions, like compactness and contractivity, are preserved under finite unions. Notice also that a fixed point $A_{*}$ of $F$ generated by $\varphi: X \multimap X$ need not be completely invariant under $\varphi$, although $\overline{\varphi\left(A_{*}\right)}=A_{*}$.

We say that a set $M$ attracts $A \subset X$ under $\varphi$ if for every $\varepsilon>0$ there exists $n_{0}$ such that $F^{n}(A) \subset \mathcal{O}_{\varepsilon} M$ for all $n \geq n_{0}$ ( $F^{n}$ denotes the $n$-fold composition of $F$ ). A minimal closed set $M$ attracting all subsets of $X$ (equivalently: attracting the whole space $X$ ) is called an attractor (see [L2]). We point out that the attractor $M$ always has the form $M=\bigcap_{n \in \mathbb{N}} F^{n}(X)$, and that our notion differs slightly from the usual concept of global attractor. (The intersection $\bigcap_{n} F^{n}(X)$ is not always an attractor: to see this, just consider the time one map for an appropriate continuous flow on a halfplane).

A good example of a condensing map is provided by multivalued weak contraction with compact values. This enables us to apply our results to weakly contractive IFS's (comp. $[\mathrm{H}],[\mathrm{W}],[\mathrm{AF}]$ ).

Let $\eta:[0, \infty) \rightarrow[0, \infty)$ be a nondecreasing right-continuous function such that $\eta(0)=0, \eta(r)<r$ for $r>0$.

A multifunction $\varphi: X \multimap X$ is a weak contraction provided there exists a function $\eta$ as above such that

$$
h\left[\varphi\left(x_{1}\right), \varphi\left(x_{2}\right)\right] \leq \eta\left(d\left(x_{1}, x_{2}\right)\right) \quad \text { for all } x_{1}, x_{2} \in X .
$$

Proposition 5. If $\varphi: X \multimap X$ is a multivalued weak contraction with compact values, then it is $\beta$-condensing.

Proof. Fix $r>\beta(A)>0$ and $\varepsilon>0$. Next, find a finite $r$-net for $A$, i.e., $\bigcup_{i} B\left(x_{i}, r\right) \supset A$. Observing that $\varphi\left[B\left(x_{i}, r\right)\right] \subset \mathcal{O}_{\eta(r)+\varepsilon} \varphi\left(x_{i}\right)$ we obtain

$$
\varphi(A) \subset \bigcup_{i} \varphi\left[B\left(x_{i}, r\right)\right] \subset \bigcup_{i} \mathcal{O}_{\eta(r)+\varepsilon} \varphi\left(x_{i}\right) .
$$

Now, put $K=\bigcup_{i} \varphi\left(x_{i}\right)$, and cover it by a finite family of balls, $K \subset$ $\bigcup_{j} B\left(z_{j}, \varepsilon\right)$. Hence we infer $\beta[\varphi(A)] \leq \eta(r)+2 \varepsilon$. Finally, since $r$ and $\varepsilon$ were arbitrary,

$$
\beta[\varphi(A)] \leq \lim _{r \rightarrow \beta(A)} \eta(r)=\eta[\beta(A)]<\beta(A) .
$$

Additionally, if $\beta(A)=0$ then $\beta[\varphi(A)]=0$, by continuity of $\varphi$. 
We will need

THEOREM 3 (on attractors of condensing maps). Let $X$ be a complete space, $\varphi: X \multimap X$ a bounded $\beta$-condensing multifunction, and $F$ the Barnsley-Hutchinson operator associated with $\varphi$. Then there exists a compact attractor $M$ and a maximal fixed point $A_{*}$ of $F$ such that $A_{*} \subset M$. If additionally $\varphi$ is uniformly Hausdorff upper semicontinuous, then $A_{*}=M$.

This improvement on [L1] and [L2] can be obtained by applying the observations made in [S] and Lemma 1.6.11 of [AKPRS].

Now, combining Theorems 3 and 2 we arrive at the following theorem which is an improvement and partial generalization of some results from [W] and $[\mathrm{K}]$.

THEOREM 4 (on attractors of compact families of condensing maps). Let $\Phi=\left\{\varphi_{t}: X \multimap X\right\}_{t} \subset \mathcal{B M}(X, X)$ be an $h_{\text {sup-compact family of bounded }}$ $\beta$-condensing multifunctions, and let $F$ be the Barnsley-Hutchinson operator associated with $\Phi$. Then $\Phi$ has a compact attractor $M$ and $F$ has a maximal fixed point $A_{*}$ such that $A_{*} \subset M$. Moreover, if all $\varphi_{t}$ are uniformly Hausdorff upper semicontinuous, then $A_{*}=M$.

Finally, note that all the results from Sections 1, 3 and 4 can be reformulated for multifunctions which are not necessarily selfmaps.

Acknowledgments. I would like to thank Professors L. Górniewicz, W. Kryszewski and D. Miklaszewski for their constructive comments.

\section{References}

[AKPRS] R. R. Akhmerov, M. I. Kamenskiǔ, A. S. Potapov, A. E. Rodkina and B. N. Sadovskiı̌, Measures of Noncompactness and Condensing Operators, Nauka, Novosibirsk, 1986 (in Russian).

[AF] J. Andres and J. Fišer, Metric and Topological Multivalued Fractals, Internat.

J. Bifur. Chaos 14 (2004), to appear.

[AG] J. Andres and L. Górniewicz, On the Banach contraction principle for multivalued mappings, in: Approximation, Optimization and Mathematical Economics, M. Lassonde, (ed.), Physica-Verlag and Springer, 2001, 1-23.

[CV] C. Castaing and M. Valadier, Convex Analysis and Measurable Multifunctions, Lecture Notes in Math. 580, Springer, Berlin, 1977.

[CoV] C. Costantini and P. Vitolo, Decomposition of topologies on lattices and hyperspaces, Dissertationes Math. 381 (1999).

[D] K. Deimling, Nonlinear Functional Analysis, Springer, Berlin, 1985.

[E] A. Edalat, Dynamical systems, measures and fractals via domain theory, Inform. and Comput. 120 (1995), 32-48.

[H] M. Hata, On some properties of set-dynamical systems, Proc. Japan Acad. Ser. A Math. Sci. 61 (1985), 99-102.

[Ha] S. Hayashi, Self-similar sets as Tarski's fixed points, Publ. RIMS Kyoto Univ. 21 (1985), 1059-1066. 
[HP] S. Hu and N. S. Papageorgiou, Handbook of Multivalued Analysis, Volume I: Theory, Math. Appl., Kluwer, Dordrecht, 1997.

[Hu] J. E. Hutchinson, Fractals and self similarity, Indiana Univ. Math. J. 30 (1981), 713-747.

[JGP] J. Jachymski, L. Gajek and P. Pokarowski, The Tarski-Kantorovitch principle and the theory of iterated function systems, Bull. Austral. Math. Soc. 61 (2000), 247-261.

[K] B. Kieninger, Iterated Function Systems on Compact Hausdorff Spaces, Berichte Math., Shaker-Verlag, Aachen, 2002.

[LM] A. Lasota and J. Myjak, Attractors of multifunctions, Bull. Polish Acad. Sci. Math. 48 (2000), 319-334.

[L1] K. Leśniak, Extremal sets as fractals, Nonlinear Anal. Forum 7 (2002), 199208.

[L2] - Stability and invariance of multivalued iterated function systems, Math. Slovaca 53 (2003), 393-405.

[S] V. Šeda, On condensing discrete dynamical systems, Math. Bohem. 125 (2000), 275-306.

[SV] J. Soto-Andrade and F. J. Varela, Self-reference and fixed points: A discussion and an extension of Lawvere's theorem, Acta Appl. Math. 2 (1984), 1-19.

[W] K. R. Wicks, Fractals and Hyperspaces, Lecture Notes in Math. 1492, Springer, Berlin, 1991.

K. Leśniak

Faculty of Mathematics and Computer Science

Nicolaus Copernicus University

Chopina 12/18

87-100 Toruń, Poland

E-mail: much@mat.uni.torun.pl

Received 2.7.2003;

received in final form 19.12.2003 